\title{
Estudio de puertas catalíticas en sensores de gas en tecnología de $\mathrm{SiC}$
}

\author{
O. CASALS M. MAFFAR ${ }^{1}$ B. BARCONES ${ }^{1}$, A. ROMANO' ${ }^{1}$, C.SERRE ${ }^{1}$, A. PÉREZ ${ }^{1}$, J.R. MORANTE ${ }^{1}$, \\ P. GODIGNON², J. MONTSERRAT², J. MILLÁN² \\ 'EME, Departament d'Electrònica, Universitat de Barcelona, 08028 Barcelona \\ ${ }^{2} I M B-C N M, C S I C ;$ Campus UAB, 08193 Bellaterra
}

\begin{abstract}
En este trabajo se presenta un estudio químico y estructural de las capas metálicas de Pt y TaSi utilizadas como puerta catalítica en sensores de gas de alta temperatura basados en dispositivos MOS de SiC. Para ello se han depositado capas de diferentes espesores sobre substratos de Si. Los resultados muestran que con la reducción del espesor de Pt y con un recocido se consigue aumentar la rugosidad de las capas de puerta, lo que debería aumentar la sensibilidad y la velocidad de respuesta de los dispositivos que las incorporasen. Otro efecto del recocido es la transformación química del material de la puerta que, para capas delgadas de $\mathrm{Pt}$ con $\mathrm{TaSi}_{x^{\prime}}$ produce la transformación total $\mathrm{Pt}$ en $\mathrm{Pt}, \mathrm{Ta}$, lo que podría afectar a las características catalíticas de la puerta. Los primeros resultados eléctricos indican que, a pesar de que las capas de Pt empleadas son gruesas y compactas, los diodos MOS túnel de $\mathrm{SiC}$ son sensibles a los gases $\mathrm{CO}$ y $\mathrm{NO}_{2}$, aunque presentan una velocidad de respuesta bastante lenta.
\end{abstract}

Palabras clave: puerta catalítica; MOS; Carburo de silicio; sensores de gas; Alta temperatura

\section{Catalitic gates for gas sensors based on SiC technology}

In this work the chemical and structural characterisation of metallic layers of $\mathrm{Pt}$ and $\mathrm{TaSi}_{\text {, }}$ employed as catalytic gates in high temperature gas sensors based on MOS devices on $\mathrm{SiC}$ substrates, is presented. For the study layers of different thickness have been deposited on $\mathrm{Si}$ substrates. The results show that the roughness of the layers increases with the reduction of the thickness of $\mathrm{Pt}$ and with annealing, which should give rise to an increase in the sensitivity and response speed of the devices. Another consequence of the annealing is the chemical transformation of the gate's materials that, for thin films of $\mathrm{Pt}$ with $\mathrm{TaSi}_{\gamma^{\prime}}$ produces the complete transformation of $\mathrm{Pt}$ into $\mathrm{Pt}_{2} \mathrm{Ta}$, which might affect the catalytic properties of the gate. The first electrical results show that, even with thick and compact Pt layers, the SiC-based MOS tunnel diodes are sensitive to $\mathrm{CO}$ and $\mathrm{NO}_{2}$ gases, although the response time is relatively long.

its response speed is too slow.

Keywords: Catalytic gate; MOS; Silicon carbide; Gas sensors; High temperature

\section{INTRODUCCIÓN}

Existe hoy en día un enorme interés en el control de los gases contaminantes emitidos a la atmósfera, lo que ha provocado un gran avance en el desarrollo de diferentes tipos de sensores de gas, entre los que destacan, por su bajo coste, los dispositivos MOS. Estos sensores utilizan una puerta catalítica porosa que disocia los gases, permitiendo, a su vez, la difusión de los productos resultantes hacia la interficie metal-óxido. La acumulación de estas cargas provoca cambios en las características eléctricas de los dispositivos. Para poder detectar estos gases es necesario que el dispositivo trabaje a alta temperatura y, en este sentido, el carburo de silicio ( $\mathrm{SiC}$ ) esta adquiriendo un gran protagonismo(1), lo que permitiría un control directo en los procesos de combustión. Además el $\mathrm{SiC}$ presenta una elevada resistencia química y física, gran conductividad térmica y el crecimiento del aislante $\left(\mathrm{SiO}_{2}\right)$ está bastante controlado, aunque, de momento la tecnología está poco desarrollada (2).

Para desarrollar estos sensores, en este trabajo se presenta un estudio químico y estructural de las diferentes capas que forman la puerta orientado a aumentar su porosidad y disminuir su espesor $\mathrm{y}$, por tanto, mejorar la respuesta del sensor.

\section{EXPERIMENTAL}

Se han fabricado diodos MOS túnel y capacidades MOS mediante la pulverización catódica de $\mathrm{Pt}$ sobre $\mathrm{SiO}_{2}$ crecido térmicamente o en ambiente de ozono (Fig.1a). Para mejorar la adherencia del Pt sobre el óxido se introduce una capa tampón de TaSi también por pulverización catódica(3). Los dispositivos se han recocido en un horno RTP a $700^{\circ} \mathrm{C}$ durante $3^{\prime}$.

Para el análisis se han preparado muestras similares sobre substrato de $\mathrm{Si}$ pero con diferentes espesores de $\mathrm{Pt}, \mathrm{TaSi}_{\mathrm{x}}$ y $\mathrm{SiO}_{2}$ (Fig.1b), con o sin recocido

\begin{tabular}{|lr|}
\hline $\mathrm{Pt}$ & $100 \mathrm{~nm}$ \\
\hline $\mathrm{TaSi}$ & $12 \mathrm{~nm}$ \\
\hline $\mathrm{SiO}_{2}$ & $1040 \mathrm{~nm}$ \\
\hline $6 \mathrm{H}-\mathrm{SiC}$ & \\
\hline
\end{tabular}

Figura 1a Dipositivos MOS Figura 1b Muestras para el análisis

La caracterización química y estructural se ha realizado mediante perfilometría interferométrica, difracción de rayos X (DRX) en incidencia rasante espectroscopía de fotoelectrones (XPS) y microscopía electrónica de transmisión (MET).

Las medidas eléctricas han consistido en la obtención de las curvas características $\mathrm{I}(\mathrm{V})$ y $\mathrm{C}(\mathrm{V})$ a diferentes temperaturas y en la medida de la variación de la tensión en condiciones de corriente constante en presencia de diferentes concentraciones de $\mathrm{CO}$ y de $\mathrm{NO}_{2}$.

\section{RESULTADOS Y DISCUSIÓN}

El análisis de las muestras depositadas y no recocidas (tabla 1) revela que no existe gran diferencia en la rugosidad de una muestra 
a otra, si bien la rugosidad relativa aumenta al disminuir el espesor. Además, la observación mediante MET de la sección transversal de uno de los diodos MOS túnel no recocidos revela que la puerta catalítica es muy uniforme, compacta y densa.

TABLA 1 EsPESORES DE LAS MUESTRAS ANALIZADAS

\begin{tabular}{|c|c|c|c|c|}
\hline Muestra & Pt (nm) & TaSix $_{\mathbf{~}(\mathbf{n m})}$ & RMS (\%) & RMS (\%) \\
\hline $\mathbf{1}$ & 100 & 24 & 6 & 6 \\
\hline $\mathbf{2}$ & 40 & 12 & 13 & 27 \\
\hline $\mathbf{3}$ & 20 & 12 & 20 & 43 \\
\hline $\mathbf{4}$ & 14 & 24 & 57 & 85 \\
\hline $\mathbf{5}$ & 20 & - & 20 & 30 \\
\hline
\end{tabular}

Las mayores diferencias estructurales se observan una vez realizado el recocido. Para la muestra con mayor espesor de Pt (muestra1) el tratamiento no provoca ningún cambio apreciable, mientras que a medida que el espesor de Pt disminuye, la rugosidad relativa aumenta considerablemente, llegándose a observar rugosidades que casi igualan el espesor nominal de la capa (muestra 4). La presencia de $\mathrm{TaSi}_{x}$ (muestra 3) en muestras recocidas también se manifiesta en un aumento adicional de la rugosidad (fig.2).

Así para la realización de sensores de gas, donde la porosidad parece ser uno de los parámetros importantes, los espesores menores de Pt, la presencia del $\mathrm{TaSi}_{\mathrm{x}}$ y el recocido parecen ser las mejores con-

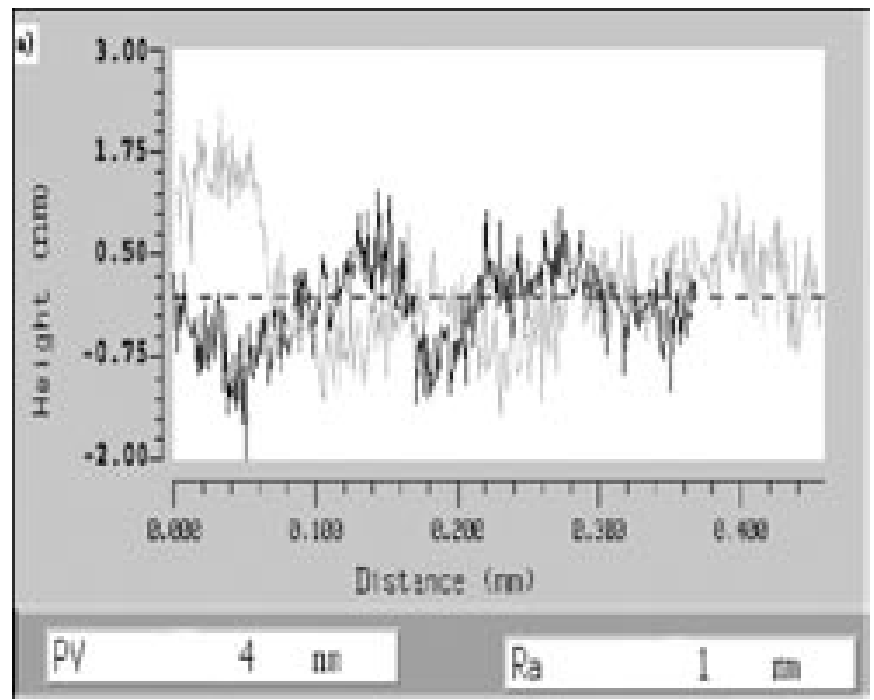

Figura 2. Muestra 3 a)antes del recocido.b) tras el recocido

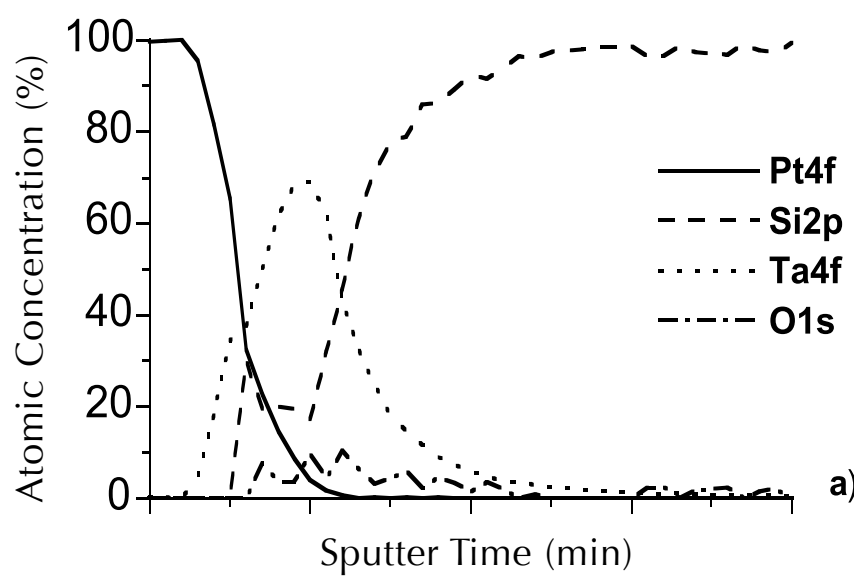

Figura 3 XPS de la muestra 3 a) sin recocer y b) recocida. diciones tecnológicas.

El análisis mediante DRX de las muestras no recocidas de la tabla 1 permiten detectar la presencia del platino metálico y sólo se observan ligeras diferencias en su textura de una muestra a otra. Los análisis por XPS de las muestras 3 (fig.3a) y 5 antes del recocido indican que las capas prácticamente no se mezclan durante el depósito, estando claramente diferenciados el Pt del TaSi $i_{x}$ en el caso de la muestra 3.

Tras el recocido se observa en la muestra 3, tanto por XPS (fig.3b) como por DRX, la transformación total del $\mathrm{Pt}$ en $\mathrm{Pt}_{2} \mathrm{Ta}$ y del tántalo restante en $\mathrm{Ta}_{2} \mathrm{O}_{5}$ y prácticamente no se detecta silicio ni oxígeno en las primeras capas. Sin embargo para muestras con espesores de $\mathrm{Pt}$ mayores se observa que, además de la formación del $\mathrm{Pt}_{2} \mathrm{Ta}$ encima del $\mathrm{SiO}_{2^{\prime}}$ en las capas superficiales persiste una gran cantidad de Pt que no ha reaccionado. El recocido de muestras delgadas $\sin \mathrm{TaSi}_{\mathrm{x}}$ (muestra 5) es completamente diferente de las anteriores, ya que el Pt reacciona con el Si para formar PtSi en la superficie.

Estos resultados indican que el recocido conlleva, además de un cambio en la rugosidad y, posiblemente, de la porosidad, un cambio químico en el material de la puerta, tanto en la capa de Pt como en la de $\mathrm{TaSi}_{x}$.

La caracterización eléctrica de los diodos MOS túnel muestra un comportamiento rectificador en los dispositivos sin recocer hasta temperaturas del orden de $200^{\circ} \mathrm{C}$, mientras que a temperaturas superiores el comportamiento es óhmico. Por el contrario, el recocido aumenta
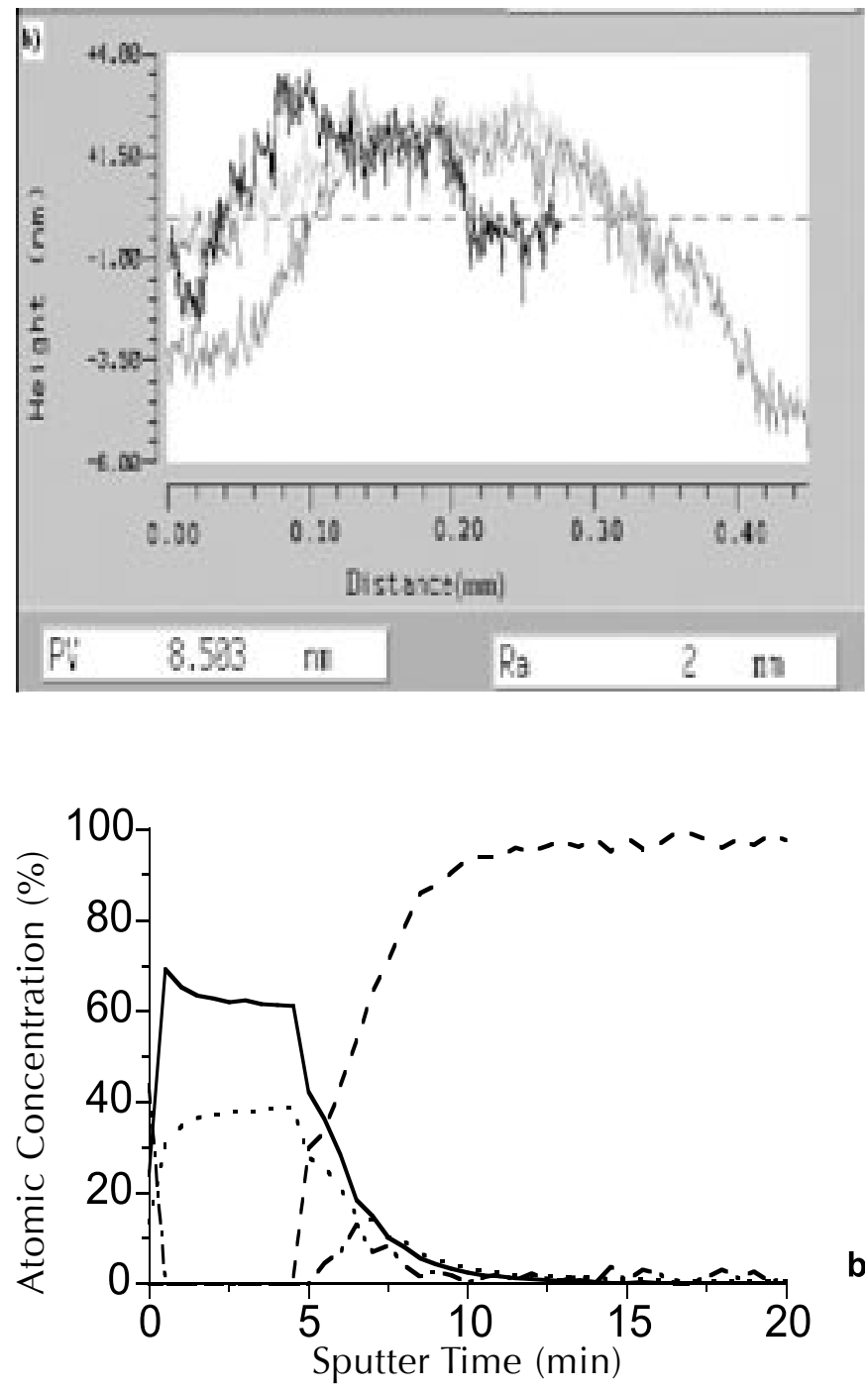


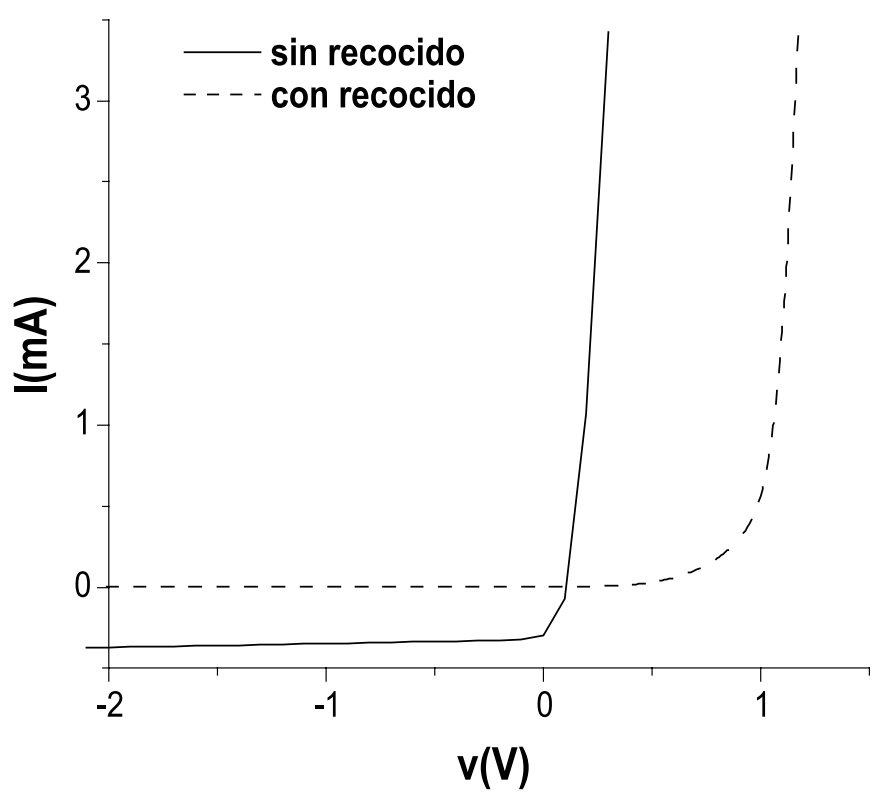

Figura $4 \mathrm{I}(\mathrm{V})$ de los diodos MOS túnel a $100^{\circ} \mathrm{C}$

el rango de temperaturas de comportamiento rectificador hasta unos $380^{\circ} \mathrm{C}$ (fig.4). Las medidas $\mathrm{C}(\mathrm{V})$ en estos diodos han permitido relacionar este comportamiento con un aumento substancial de la altura de barrera.

La respuesta de los diodos MOS túnel ante la presencia de diferentes concentraciones de gases $\mathrm{CO}$ (fig.5) y $\mathrm{NO}_{2}$ se manifiesta en una variación de la tensión en los bornes del dispositivo que depende directamente de la concentración del gas. En concreto, para el gas $\mathrm{CO}$, la variación de hasta $1,3 \mathrm{mV}$ a la temperatura óptima de trabajo de $250^{\circ}$, mientras que en presencia de $10 \mathrm{ppm}$ de $\mathrm{NO}_{2}$ y a esta temperatura (no óptima para este gas) se observa una variación de $100 \mu \mathrm{V}$. En ambos casos el tiempo de respuesta del dispositivo es relativamente largo, de centenares de segundos.

Tal como se ha mencionado anteriormente, los dispositivos sensores de gas fabricados tienen una puerta de metal catalítico gruesa y compacta, que podría justificar su reducida sensibilidad a la presencia de gas, así como el largo tiempo de respuesta, ya que las especies químicas disociadas en la superficie tienen grandes dificultades para difundir hacia la interficie entre el metal y la capa de $\mathrm{SiO}_{2}$.

Con los resultados obtenidos del análisis químico y estructural se propone la fabricación de un nuevo conjunto de dispositivos sensores que utilicen un aislante $\left(\mathrm{SiO}_{2}\right)$, una capa tampón de $\mathrm{TaSi}_{x}$ y la puerta catalítica de Pt delgada, de unos 20-40 nm, seguido de un recocido. De todas maneras hay que controlar si, al utilizar estas capas de Pt tan delgadas, la transformación completa del Pt pueda afectar a la disociación catalítica en la superficie de la puerta.

\section{CONCLUSIONES}

En este trabajo se ha presentado un estudio químico y estructural de las capas metálicas utilizadas como puerta catalítica en sensores de gas basados en dispositivos MOS. El estudio ha mostrado que los

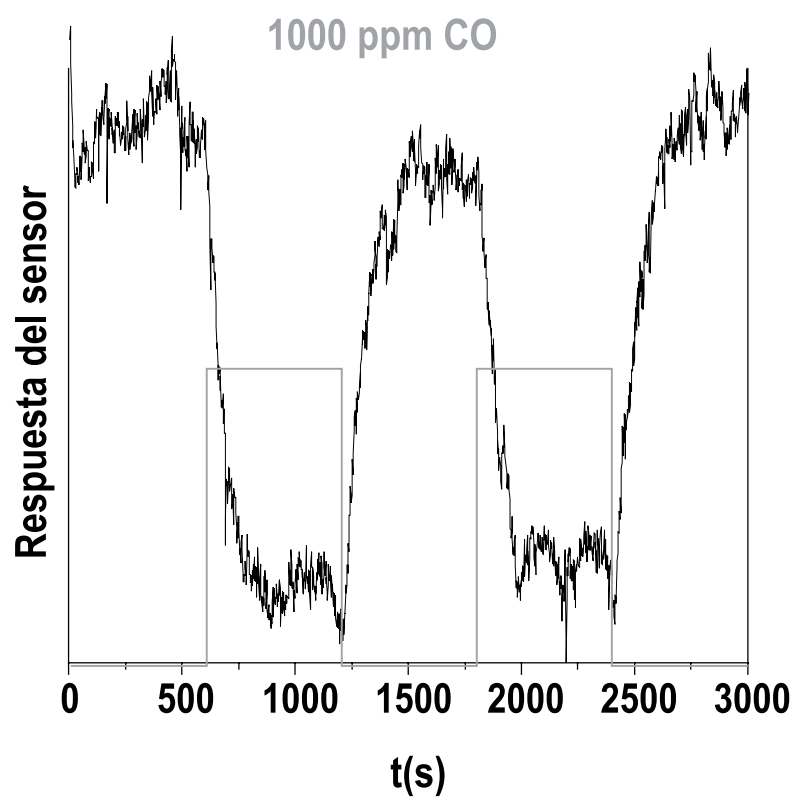

Figura 5 Respuesta de un diodo túnel a $1000 \mathrm{ppm}$ de $\mathrm{CO}$ a $250^{\circ} \mathrm{C}$ a una intensidad constante de $10 \mu \mathrm{A}$

espesores más reducidos de Pt parecen dar la mayor rugosidad y, por tanto, porosidad, sobre todo después del recocido en un horno RTP, parámetros importantes para aumentar la sensibilidad y reducir el tiempo de respuesta de estos dispositivos. Se ha observado, también, que el recocido conlleva cambios químicos en los materiales de la puerta que benefician las propiedades eléctricas de los dispositivos con puertas gruesas, pero que podrían tener efectos negativos cuando se utilizasen capas de Pt delgadas. Finalmente, se han presentado los primeros resultados de la respuesta eléctrica de los dispositivos sensores, mostrando que, incluso en estos primeros dispositivos, ya presentan cierta sensibilidad, que debería poderse mejorar con el uso de capas optimizadas.

\section{AGRADECIMIENTOS}

Este trabajo ha sido financiado en parte por la Subdirección General de Proyectos de Investigación del Ministerio de Ciencia y Tecnología, a través del proyecto TIC2000-1403-C03.

\section{BIBLIOGRAFÍA}

1. A. Lloyd-Spetz, A. Baranzahi, P. Tobias, I. Lundström. «High temperature sensors based on metal-insulator-silicon carbide devices» Phys. Stat. Sol.(a), 162, 493-510 (1997).

2. J. A.. Cooper, Jr. «Advances in SiC MOS technology» Phys. Stat. Sol.(a), 162, 305-320 (1997).

3. L. Uneus, P. Ljung, M. Mattson, P. Martesson, R. Wigren, P. Tobias, I. Lundström, L.-G. Ekedahl, A.Lloyd-Spetz. «Measurements with MISiC and MOS sensors in flue gases» Proc. Eurosensors XIII, La Haya (Holanda), septiembre 12-15,521-524(1999).

Recibido: 1.2 .03

Aceptado: 30.11 .03 